\title{
Traditional chinese medicine in treating IgA nephropathy: from basic science to clinical research
}

\author{
Xin-hui Wang, ${ }^{1,2}$, Rui Lang ${ }^{1}$, Ying Liang ${ }^{1}$, Qin Zeng ${ }^{1}$, Nan Chen ${ }^{1}$, Ren-huan Yu ${ }^{1, *}$ \\ 'Xiyuan Hospital, China Academy of Chinese Medical Sciences, Beijing, China; \\ ${ }^{2}$ Graduate School of China Academy of Chinese Medical Sciences, Beijing, China;
}

\section{ABSTRACT}

IgA nephropathy (IgAN) is a major cause of chronic kidney disease (CKD) and end-stage renal disease worldwide. Currently, clinical interventions for IgAN are limited, and many patients seek out alternative therapies such as traditional Chinese medicine (TCM). In the last several years, TCM has accumulated ample application experiences and achieved favorable clinical effects. This article summarizes high-quality research from basic science to clinical applications aimed to provide more evidence-based medicine proof for the clinical treatment of IgAN. In summary, qi and yin deficiency accounted for the largest proportion in IgAN patients, and the treatment of IgAN should be based on supplementing qi and nourishing yin. Further, for patients with severe IgAN, the treatment combination of Chinese and Western medicines is better than pure Chinese medicine or hormone therapy. In addition, the pharmacological mechanism of Chinese herbal medicines is mostly based on restoring the immune function, relieving the inflammation damage, and inhibiting proliferation of the glomerular mesangial cells.

Key words: IgA nephropathy, Traditional Chinese Medicine, treatment, herbal medicine, review

\section{INTRODUCTION}

IgA nephropathy (IgAN) is the mostcommon pattern of primary glomerulonephritis worldwide. It is characterized by repeated episodes of gross or microscopic hematuria and often proteinuria. ${ }^{[1]}$ Within 20 years of diagnosis, nearly $25 \%-30 \%$ of IgAN patients will irreversibly lead to end-stage renal disease (ESRD) ${ }^{[2]}$ In China, IgAN accounts for $52.66 \%$ of the cases in primary glomerulonephritis. ${ }^{[3]}$ The Kidney Disease: Improving Global Outcomes (KDIGO) 2020 guideline recommends that the management of IgAN should focus on supportive care. ${ }^{[4]}$ For patients with persistent proteinuria and at risk of renal function progression, it is still controversial whether the use of hormones/ immunosuppressants is beneficial. ${ }^{[5-7]}$ The current treatment for IgAN remains insufficient and challenging.

Over the past few decades, traditional Chinese medicine (TCM) has been widely used for the prevention and treatment of kidney disease and was supported by series of evidence-based medicine for the effectiveness and safety. ${ }^{[8-11]}$ TCM treatment based on syndrome differentiation is multitarget and multipathway, which can achieve its unique therapeutic effect by adjusting the yin and yang of the human body system. ${ }^{[12]}$ Herein, we summarize the impacts of TCM in treating IgAN from a theoretical basis to clinical applications and experimental study.

\section{THEORETICAL BASIS OF IGA NEPHROPATHY TREATED BY TRADITIONAL CHINESE MEDICINE}

According to several multicenter surveys, most scholars considered that qi and yin deficiency accounted for the largest proportion in IgAN patients and proposed that the treatment of IgAN should be based on reinforcing qi and replenishing yin. ${ }^{[13-15]}$ 
The study results of 467 patients with IgAN showed that qi and yin deficiency accounted for $66.81 \% \cdot{ }^{[16]}$ Two other studies collected data of 1016 and 1014 IgAN patients, respectively, and also came to the same conclusion. ${ }^{[17-18]}$ When course prolonged, the qi and yin deficiency will be aggravated and lead to yin deficiency of liver and kidney, then progressed to yang deficiency of spleen and kidney. ${ }^{\left[{ }^{[1]}\right]}$ Many studies used Lee's grading system and Katafuchi score system to classify the severity of glomerulus and tubulointerstitial lesions of $\operatorname{IgAN}$ and then to investigate the relationship between renal pathological classifications and TCM syndromes. ${ }^{[2-21]}$ It is reported that the pathological change of IgAN patients with the spleen and kidney yang deficiency syndrome was the most serious, and follow by the liver and kidney yin deficiency syndrome. ${ }^{[22]}$ For IgAN patients with Lee's grade above, conventional treatment is nourishing yin of liver and kidney or nourishing yang of spleen and kidney.

In recent years, the observation of visible hematuria in IgAN patients induced by respiratory infections has been taken as evidence of the "four-hit" hypothesis in the etiology mechanism of $\operatorname{IgAN} .{ }^{[23]}$ The occurrence of $\operatorname{IgAN}$ is associated with tonsillitis, which enhances the polymeric galactose-deficient IgA1 (Gd-IgA1) production via IgA class switching and immuno-activation. ${ }^{[24]}$ The tonsillectomy has been one of the hotspots in $\operatorname{IgAN}$ therapy, but the results of studies from Asia and Europe drew diametrically opposite conclusions. ${ }^{[25-28]}$ The data from several cross-sectional studies in China showed that in IgAN patients, the chronic pharyngitis is around $62 \%-64 \%$, and patients who had two or more respiratory mucosal symptoms accounted for $66.7 \% .^{[29-30]}$ Chronic pharyngitis may be the limited factor for the effectiveness of tonsillectomy in patients with IgAN, which can be treated by TCM with obvious advantage.

\section{LEADING CLINICAL STUDIES ON TREATING IGA NEPHROPATHY WITH TRADITIONAL CHINESE MEDICINES}

\section{Chinese herbal compounds focusing on reinforcing qi and replenishing yin}

Professor Nie Lifang built up the "Yiqizishen Granules" (Huang Qi [Astragalus membranaceus], Tai Zi Shen [Radix pseudostellariae], Sheng Di [Radix rehmanniae], Xiao Ji [Herba cirsii], Jin Yin Hua [Flos lonicerae], Han Lian Cao [Herba eclipta], Zhi Zi [Fructus gardeniae], Dang Gui [Angelica sinensis], Qian Shi [Gorgon fruit]) to treat IgAN. A multicenter, randomized, parallel controlled trial focuses on evaluating the effect of Yiqizishen Granules on hematuria. A total of 264 patients were randomly classified into the treatment group (Yiqizishen Granules) and the control group (Shenyan Kangfu Tablets). After 12 weeks of treatment, the detection rate of hematuria was $13.73 \%$ in the treatment group and was $27.52 \%$ in the control group $(P<0.05) \cdot{ }^{[31]}$ Another randomized controlled clinical study used Yiqizishen capsule to treat $176 \mathrm{IgAN}$ patients with proteinuria. After 12 weeks of treatment, the decrease of the 24-hour urinary protein quantification is $42.6 \%$ in the treatment group and $32.6 \%$ in the control group $(P<0.05) \cdot{ }^{[32]}$ Chen et al. conducted a multicenter, double-blind, double-simulation, randomized controlled trial to evaluate the therapeutic effect of Shenhua Tablets (Huang Qi [Astragalus membranaceus], Nv Zhen Zi [Ligustrum lucidum ait], Bai Zhu [Atractylodes macrocephala], Jin Yin Hua [Flos lonicerae]). A total of $131 \mathrm{IgAN}$ patients with qi and yin deficiency syndrome were included. At 12 weeks of treatment, both Shenhua tablets and Fosinopril could reduce urine protein quantification, and there were no differences between the groups. ${ }^{[3]}$

A randomized controlled study conducted by $\mathrm{He}$ et al. included $79 \mathrm{IgAN}$ patients with qi and yin deficiency. The control group was treated with "Guben Tongluo Prescription” (Huang Qi [Astragalus membranaceus], Dan Shen [Salvia miltiorrbiza], Tao Ren [Peach kerne], Nv Zhen $\mathrm{Zi}$ [Ligustrum lucidum ait], Han Lian Cao [Herba eclipta], Gui Jian Yu [Euonymus alatus], Bai Mao Gen [Rhizoma imperata]). On this basis, the treatment group added Chinese medicines for clearing heat and relieving sore throat, which included Chan Tui [Cicada slough], Jie Geng [Platycodon grandiflorum], Jiang Can [Bombyx batryticatus], and Xuan Shen [Radix scropbulariae]. After 8 weeks of treatment, the total effective rate of the treatment group was significantly higher than that of the control group $(85 \%$ vs. $60 \%) \cdot{ }^{[34]}$ A prospective randomized controlled clinical study by Yu et al. applied the "Yiqi Yangyin Liyan Granules" (Huang Qi [Astragalus membranaceus], Fang Ji [Stephania tetrandra], He Ye [Lotus leaf], Huang Jing [Polygonatum sibiricum], Jin Yin Hua [Flos lonicerae], Chan Tui [Cicada slough], Zi Su Ye [Perilla leaves]) combined with knife pecking method for treating IgAN patients accompanied with pharyngeal inflammation. Ninety patients were randomly divided into two groups: 45 in the therapeutic group and 45 in the control group. The patients in the therapeutic group received Yiqi Yangyin Liyan Granules orally twice daily and pharyngeal knife pecking therapy twice a week, and the patients in the control group received Irbesartan $150 \mathrm{mg}$ orally daily. After 8 weeks of regular treatment, patients were followed for additional 24 weeks, and the total effective rate of the therapeutic group was $72.1 \%$, which was significantly higher than the control group. ${ }^{[35]}$

A multicenter, randomized, placebo-controlled study was conducted by Wang et al., and it aimed to observe the clinical efficacy of "Chai Qin Recipe" (Chai Hu [Radix bupleuri], 
Huang Qin [Radix scutellariae], Dang shen [Radix codonopsis], Huang Qi [Astragalus membranaceus], Ban Zhi Lian [Scutellaria barbata], Bai Hua She She Cao [Hedyotis diffusa], Long Kui [Solanum nigrum]) for IgAN treatment. In the study, a total of 79 cases were divided into control group (valsartan 80 $\mathrm{mg}+$ placebo) and test group (valsartan $80 \mathrm{mg}+$ Chai Qin Recipe). After 6 months of treatment, the total effective rate in the test group was better than that in the control group $(77.5 \%$ vs. $56.4 \%)$. $^{[36]}$

\section{Chinese herbal compounds focusing on dispelling wind and eliminating dampness}

Chen et al. conducted a multicenter, randomized, placebocontrolled study to observe the clinical efficacy of "Qu Feng Shi Recipe” (Huang Qi [Astragalus membranaceus], Dang Gui [Angelica sinensis], Tao Ren [Peach kerne], Ji Xue Cao [Centella asiatica]) for treating IgAN patients with wind-damp syndrome. A total of 174 patients completed the program: 96 patients in the treatment group (Qu Feng Shi Recipe + Tripterysium glycosides) and 78 patients in the control group (placebo). After 12 weeks of treatment, in both groups, 24-hour urinary protein quantity and serum creatinine were reduced and glomerular filtration rate (eGFR) was increased; however, the overall improvement was seen in the treatment group compared with the control group $(P<0.05)$. ${ }^{[37]}$

A retrospective study conducted by $\mathrm{Yu} e t$ al. included 356 patients treated with Chinese herbal medicines for dispelling wind dampness. The follow-up endpoint of patients was defined as entering ESRD or the eGFR decreased by $50 \%$ compared with the renal puncture. The median follow-up time was 5.33 years, and 87 patients were lost to follow up. The 5-year renal survival rate of 219 patients was 98\%. Further, 219 patients were divided into group A (49 cases), who had treatment with Chinese herbal medicines for dispelling wind dampness, and group B (170 cases) who had combined treatment with Chinese herbal medicines and hormones/immunosuppressants for dispelling wind dampness. There was no significant difference in total effective rate between the two groups $(89.8 \%$ vs. $88.8 \%)$, and the complete remission rate was higher in group $\mathrm{A}(55.1 \%$ vs. $31.8 \%){ }^{[38]}$ Another study from Hong Kong used ESRD as the endpoint and included 168 patients treated with Western medicine. The 5-year renal survival rate was $85.9 \% .{ }^{[39]}$ This shows that the long-term effect of using Chinese herbal medicine for dispelling wind dampness combined with Western medicine is better than pure Western medicine treatment.

\section{Chinese herbal compounds focusing on treatment of severe IgA nephropathy}

A prospective, multicenter, randomized, placebocontrolled clinical trial by He Liqun's group evaluated the efficacy and safety of "Buyi Ganshen Recipe" (Sheng Di
[Radix rehmanniae], Shan Yu Rou [Pulp of cornus], Gou Qi Zi [Fructus lyci], Nv Zhen Zi [Ligustrum lucidum ait], Han Lian Cao [Herba eclipta]) combined with losartan in the treatment of IgAN patients with Lee's grade above and with yin deficiency of liver and kidney. In the study, a total of 156 cases were included and randomized into the treatment group (Buyi Ganshen Recipe + losartan $100 \mathrm{mg}$ ) and the control group (placebo + losartan $100 \mathrm{mg}$ ). The duration of treatment was 24 weeks. The total effective rate in the treatment group was higher than that in the control group (70.67\% vs. $38.57 \%$ ). ${ }^{[40]}$

Another randomized, double-blind, placebo-controlled, multicenter clinical trial conducted by Deng et al. aimed to investigate the efficacy and safety of "Zibu Ganshen Granules" (Sheng Di [Radix rehmanniae], Huang Jing [Polygonatum sibiricum], Nv Zhen Zi [Ligustrum lucidum ait], Fu Ling [Poria cocos], Bai Shao [Radix paeonia alba], Dang Gui [Angelica sinensis], Shan Yu Rou [Pulp of cornus], Zi Hua Di Ding [Herba violae], Bai Zhu [Atractylodes macrocephala]) plus oral prednisone. In the study, 67 IgAN patients with Lee's grade above and with yin deficiency of liver and kidney were divided in the integrative medicine group (Zibu Ganshen Granules + oral prednisone) and the control group (placebo + oral prednisone). At the end of 48-week follow-up, the eGFR level was increased more from the baseline in the integrative medicine group than in the control group $(P<0.05) \cdot{ }^{[41]}$

There is also an earlier study which is randomized, controlled, and double-blind multicenter trial by Yueyi Deng team conducted to investigate the efficacy of "Jianpi Bushen Tongluo Granules" (Huang Qi [Astragalus membranaceus], Huang Jing [Polygonatum sibiricum], Du Zhong [Bark of eucommia], Chuan Xiong [Rhizoma chuanxiong], Ge Gen [Radix puerariae]). A total of 120 IgAN patients (Lee's grade III and above) were recruited, and all the patients presented with the syndrome of yang deficiency of spleen and kidney. The test group was given Jianpi Bushen Tongluo Granules plus prednisone, while the control group was given placebo plus prednisone. The eGFR in both test and control groups showed an increasing trend compared with baseline in 48 weeks, while the treatment group increased significantly. ${ }^{[42]}$

In conclusion, for patients with severe IgAN (Lee's grade and above), the combination of Chinese and Western medicines is better than pure Chinese medicine or hormone therapy.

\section{Single Chinese herbs for treating of IgA nephropathy}

Abelmoschus manihot, also known as "Huangkui" in Chinese, is extracted from Flos Abelmoschus Manihot. ${ }^{[43]}$ Its proven considerable clinical significance is mainly improving 
renal function and reducing proteinuria in patients with

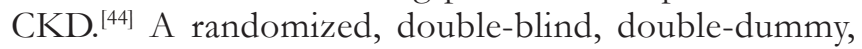
multicenter trial was conducted to evaluate the efficacy of the Huangkui capsule. A total of 89 hospitals participated with the completion of 1470 patients. The treatment group was given Huangkui capsule $2.5 \mathrm{~g}$ per day, and the control group was given losartan potassium $100 \mathrm{mg}$ per day, and they were followed up for 48 weeks. Results indicated that the 24-hour protein urinary quantification decreased by $230 \mathrm{mg}$ and $253 \mathrm{mg}$ in the Huangkui capsule and losartan potassium groups, respectively. The average decrease of the eGFR was $0.41 \mathrm{ml} / \mathrm{min} / 1.73 \mathrm{~m}^{2}$ and $0.76 \mathrm{ml} / \mathrm{min} / 1.73 \mathrm{~m}^{2}$, respectively. From the evidence, the treatment effect of the Huangkui capsule was not inferior to losartan at $100 \mathrm{mg}$ per day. ${ }^{[45]}$

Tripterygium wilfordii Hook. f. (TwHF) was recognized as an essential traditional medicinal plant, which have been frequently used to treat a variety of inflammatory and autoimmune diseases, such as systemic lupus erythematosus, nephritis, and rheumatoid arthritis. ${ }^{[4]}$ A prospective cohort study was conducted to evaluate the efficiency and side effect profiles of TwHF (especially double-dose 120 $\mathrm{mg} / \mathrm{d}$ ) in IgAN patients with heavy proteinuria. Forty-nine patients were divided into three groups: the patients in the prednisone (PRE) group received prednisone $1 \mathrm{mg} / \mathrm{kg}$ daily, those in the conventional-dose TwHF (CTW) group received TwHF $1 \mathrm{mg} / \mathrm{kg}$ daily, and those in the doubledose TwHF (DTW) group received TwHF $2 \mathrm{mg} / \mathrm{kg}$ daily. The complete remission rate of the DTW group was the highest $(87.5 \%)$ and followed by the PRE group (69.2\%). ${ }^{[4]}$ In addition, no serious adverse events were observed in the patients in the DTW group.

\section{LEADING MECHANISM STUDIES ON TREATING IGA NEPHROPATHY WITH TRADITIONAL CHINESE MEDICINES}

\section{Immunomodulation effects of Traditional Chinese Medicines}

The most prominent feature of $\mathrm{IgAN}$ is the deposition of Gd-IgA1 immune complexes in the mesangial. ${ }^{[48]}$ When the mucosal immune system was affected by pathogen infections, the aberrant Gd-IgA1 antibodies will be produced by $\mathrm{B}$ cells, and then combined with specific $\mathrm{IgG}$ antibodies to form immune complex deposits in the mesangial area. ${ }^{[4-50]}$ The interplay between the mucosalderived antigens with $\mathrm{B}$ cells has been confirmed to activate via T-cell-dependent and T-cell-independent pathways. ${ }^{[51]}$ Several traditional medicines and natural medicinal monomers have been reported to regulate the balance of immune function. ${ }^{[52-53]}$ The main ingredients of TwHF are total alkaloids and Terpenoid lactones. ${ }^{[54]}$ Ren et al. confirmed that Terpenoid lactone therapy can reduce the levels of Gd-IgA1 in peripheral blood and balance the proportion of Treg in CD4+T cells in peripheral blood mononuclear cells. Hesperidin is a kind of flavonoid compound that has exact pharmacology activity and versatility. ${ }^{[5]}$ Chen et al. have found that Hesperidin could ameliorate the renal function in rats with $\operatorname{IgAN}$, and the mechanism may be related to the reduction of the expression of JAK/STAT signaling pathway-related protein STAT3. The JAK/STAT signaling pathway has a key role to play in the differentiation of CD4+T lymphocytes. ${ }^{[5]}$ TLR4, renowned for its functions in recognizing pathogen-associated molecular patterns in innate immunity, is increased in patients with $\operatorname{IgAN}$ and correlated with the pathogenesis of IgAN. Rhein, an acidic compound derived from rbubarb, has been found to inhibit TLR4 and TGF- $\beta 1$ expressions in IgAN rats. ${ }^{[57]}$

The treatment of the "Yiqi Yangyin Liyan Formula" combined with the knife pecking method achieved significant clinical efficacy for IgAN patients. Further studies confirmed that this treatment could regulate subsets of $\mathrm{T}$ cells in the peripheral blood of IgAN patients. After 24 weeks of treatment, the proportion of CD4+ in peripheral blood was significantly reduced. ${ }^{[58]}$ Another study found that "Gouqi Fushen Pills" reduced the level of hematuria and proteinuria in IgAN rats via correct the Th1/Th2 ratio drift. ${ }^{[5]}$

\section{Inhibition of renal fibrosis and inflammatory injury}

Inflammation damage can accelerate the progression of IgAN by affecting the function of T cells, activating complement, and stimulating the body to produce autoimmune antibodies. ${ }^{[0]}$ As a traditional Chinese compound formula, Zhen-wu-tang (ZWT) is composed of five herbs (Fu Zi [Aconitum carmichaelii debeaux], Fu Ling [Poria cocos], Bai Zhu [Atractylodes macrocephala koidz], Bai Shao [Paeonia lactiflora pall, Sheng Jiang [Zingiber officinale roscoe]). The study by Liu et al. found that ZWT could ameliorated podocyte injury in IgAN via upregulation PPAR $\gamma$ expression, and the potential underlying mechanism might involve the inhibition of the NF- $x \mathrm{~B}$ pathway. ${ }^{[61]}$ Further, Li et al. indicated that in human renal tubular epithelial cells (HK-2) induced by lipopolysaccharide (LPS), ZWT-contained serum was able to dramatically block NF$x \mathrm{~B}$ nuclear translocation and activate the expression of the NLRP3 inflammasome, and then led to the decreased expression of IL-1 and caspase-1. ${ }^{[62]}$

Furthermore, many herbs have active ingredients that may improve IgAN inflammatory factors and regulate inflammatory pathways excellently. In IgAN rats, Periostracum cicadae could result in the reduction of the levels of TNF- $\alpha$, IL-1 $\beta$, and IL- 6 in serum. ${ }^{[63]}$ In addition, treated 
with hirudin, the expression of fibrosis indexes in IgAN rat kidney, included TGF- $\beta 1$, collagen-IV, and fibronectin-1, was remarkably suppressed. ${ }^{[64]}$ Schisandrin B therapy significantly increased the expression of cytoplasmic p65 and NF- $x \mathrm{~B}$ in LPS-induced HK-2 cells. ${ }^{[65]}$

\section{Inhibition of the proliferation of glomerular mesangial cells}

The proliferation of glomerular mesangial cells (GMCs) and the secretion of extracellular matrix (ECM) are essential pathological changes leading to glomerular sclerosis. ${ }^{[6]}$ Saikosaponin-d (SSd) is extracted from a traditional herb in China, Chai Hu (radix bupleur). In vitro experiments, SSd could inhibit the proliferation of GMCs and the synthesis of ECM proteins via downregulating the expression of CDK4, c-Jun, and c-Fos genes. ${ }^{[67]}$ Kunxian capsules are composed of Hai Tang (Kunming mountain begonia), Yin Yang Huo (Herba epimedii), Gou Qi Zi (Fructus lycii), and Tu Si Zi (Semen cuscutae). Zeng et al. found that Kunxian capsules could significantly alleviate diffuse proliferation of GMCs and stroma and deposition of immune complexes in IgAN rats. ${ }^{\left[{ }^{[8]}\right]} \mathrm{Ji}$ Xue Cao (Centella asiatica) is increasingly and widely used in the treatment of many diseases, such as scar healing, type 2 diabetes, and cardiovascular diseases. Asiaticoside is the main active component of Ji Xue Cao. A vitro study confirmed that asiaticoside inhibited proliferation of GMCs via the upregulation of Mfn2 and the inhibition of Ras-Raf-ERK/MAPK. ${ }^{[69]}$

\section{CONCLUSIONS}

$\operatorname{IgAN}$, characterized by $\operatorname{Ig} \mathrm{A}$ deposition in the glomerular mesangium, is not a benign disease. Although the best treatment of IgAN remains to be defined, the Western medicine mentioned included prednisolone, immunosuppressants, angiotensin-converting enzyme inhibitors (ACEIs), and angiotensin receptor blockers (ARBs) which can improve IgAN symptoms to a certain extent and control the disease. ${ }^{[4]}$ For those patients with persistent proteinuria $1 \mathrm{~g}$ per day and eGFR $>50 \mathrm{ml} /$ $\mathrm{min} / 1.73 \mathrm{~m}^{2}$, high-dose corticosteroid therapy is suggested. However, both the efficacy for kidney function preservation and the safety for serious treatment-related severe events have evoked the attention of the use of immunosuppressive therapy in IgAN patients. ${ }^{[5-7]}$ Chinese medicine emphasized the overall concept in treating disease, and the TCM mainly produced efficacy through TCM syndrome differentiation. The most common TCM syndrome type found in IgAN patients was qi and yin deficiency. ${ }^{[13-14,16-18]}$ With the damaged degree of pathological damage in IgAN patients increases gradually, TCM syndrome manifests changes from deficiency qi and yin to deficiency yin of liver and kidney, and then deficiency yang of spleen and kidney. ${ }^{[19-21]}$ Besides, the stasis syndrome and rheumatism syndrome are significant comorbidities in the process of IgAN chronic progression. ${ }^{[18]}$ The characteristics of TCM treatment for IgAN are mainly composed of tonic deficiency medicines, supplemented by blood-activating and stasis-eliminating medicines, heat-clearing medicines, inducing diuresis and excreting dampness medicines, and astringent medicines to combine with formulas.

Currently, Chinese herbal compounds that are based on the basic theory of TCM and syndrome differentia achieved the remarkable curative effects in clinical experiments. More and more TCM has been used for the prevention and treatment of IgAN and gradually approved worldwide. TCM also has shown promising effects with regard to safety and renoprotection in some prospective, multicenter, randomized, controlled clinical trial conducted on IgAN patients. For IgAN patients with a small amount of proteinuria (less than $3.5 \mathrm{~g} / 24$ h), benefiting qi and nourishing yin treatment to improve the clinical symptoms such as hematuria, proteinuria, and edema is superior to ACEI or ARB treatment, and there is no risk of hypotension. ${ }^{[31-32]}$ Besides, added herbs for relieving sore throat based on benefiting qi and nourishing yin to treat IgAN can achieve a better clinical effect that the total effective rate reached about $80 \% \cdot{ }^{[34-35]}$ For IgAN patients with Lee's grade above III, both nourishing yin of liver and kidney and invigorating yang of spleen and kidney can effectively reduce the clinical symptoms, relieve the side effects of hormones and immunosuppressants, and delay the disease progression. ${ }^{[40-42]}$ And what's more, Chinese medicine combined with hormone therapy compared with the Chinese medicine or hormone therapy alone achieved the better curative effect in severe IgAN patients. TCM is worth to generalizing and apply as a treatment method.

In the last decades, several natural medicinal monomers have been widely used in the treatment of IgAN and are known to regain the balance of immune function, ${ }^{[55-59]}$ regulate the expression of inflammatory factors, ${ }^{[61-65]}$ and inhibit the proliferation of GMCs. ${ }^{[67-69]}$ Among these, the Terpenoid lactones extracted from TwHF have been widely used as an alternative to immunosuppressive medications for the treatment of primary glomerular disease. ${ }^{[55,70-71]}$ But the multitarget mechanisms explaining the efficacy of Chinese herbal compounds on IgAN have not been fully clarified. It is widely known that the therapeutic effect of Chinese medicines results from synergistic interactions by multiple ingredients. ${ }^{[72]}$ Thus, to truly research Chinese medicines, systematic biological methods should be established, and all the elements of herbs or formulae in TCM should be taken into consideration simultaneously.

Moreover, despite the recent progress in applying TCM 
to the treatment of $\operatorname{IgAN}$, the number of multicenter, large-sample, randomized clinical trials on this topic is still insufficient. With future research on the curative effect of TCM in patients in IgAN, the establishment of an efficacy evaluation system under modern scientific methods may also be required.

\section{Source of Funding}

This study was supported by the Research project of Haidian District Health Commission, Beijing, China (HP2021-50-50401).

\section{Conflict of Interest}

None declared.

\section{REFERENCES}

1. Lai KN, Tang SW, Schena FP, Novak J, Tomino Y, Fogo A, et al. IgA nephropathy. Nat Rev Dis Primers 2016; 2: 16001.

2. Rodrigues JC, Haas M, Reich HN. IgA Nephropathy. Clin J Am Soc Nephro 2017; 12: 677-86.

3. Hou JH, Zhu HX, Zhou ML, Le WB, Zeng CH, Liang SS, et al. Changes in the Spectrum of Kidney Diseases: An Analysis of 40,759 Biopsy-Proven Cases from 2003 to 2014 in China. Kidney Dis (Basel) 2018; 4: 10-9.

4. KDIGO clinical practice guidelines on glomerular diseases (2020) Chapter 2: immunoglobulin A nephropathy. Kidney Int Suppl 2020; 6: 109-33.

5. Rauen T, Eitner F, Fitzner C, Sommerer C, Zeier M, Otte B, et al. Intensive Supportive Care plus Immunosuppression in IgA Nephropathy. N Engl J Med 2016; 373: 2225-36.

6. Lv JC, Zhang H, Wong MG, Jardine MJ, Hladunewich M, Jha V, et al. Effect of oral methylprednisolone on clinical outcomes in patients with IgA nephropathy: the testing randomized clinical trial. JAMA 2017; 318: 432-42.

7. Hou JH, Le WB, Chen N, Wang WM, Liu ZS, Liu D, et al. Mycophenolate mofetil combined with prednisone versus full-dose prednisone in IgA nephropathy with active proliferative lesions: a randomized controlled trial. Am J Kidney Dis 2016; 69: 788-95.

8. Zhong YF, Menon M, Deng YY, Chen YY, He JC. Recent advances in traditional Chinese medicine for kidney disease. Am J Kidney Dis 2015; 66: 513-22.

9. An P, Dong S, Li XY, Cai ZM, Ye BY, Zhang AJ, et al. Wenyang Huazhuo Fang exerts transient receptor potential cation channel subfamily $\mathrm{C}$ member-dependent nephroprotection in a rat model of doxorubicininduced nephropathy. J Tradit Chin Med 2020; 40: 613-20.

10. Xia XC, Mao DX, Dai HM, Wu X,Zhang ZY, Wang HY,et al.Effect of Cyclocarya paliurus polysaccharides on streptozotocin-induced diabetic nephropathy in rats. J Tradit Chin Med 2020; 40: 956-64.

11. Song ZL, Qin TY, Pan YJ, Hua Q, Wu LL, Liu TH. Shenkang injection improves coagulation in patients with chronic kidney disease: a systematic review and Meta-analysis. J Tradit Chin Med 2019; 39:451-8.

12. Zhong YF, Deng YY, Chen YP, Chuang P, He CJ. Therapeutic use of traditional Chinese herbal medications for chronic kidney diseases. Kidney Int 2013; 84: 1108-18.

13. Nie LF, Yu DJ, Yu RH, Lin XB, Sun JS, Zhang SR, et al. Multicenter prospective study on TCM syndrome distribution of 308 cases of IgA nephropathy. BeiJing Zhong Yi Yao Da Xue Xue Bao 2005; 28: 66-8.

14. Nie LF, Han DY, Yu RH, Yu DJ, Sun HY, Wang HX, et al. Distribution of Qi and yin deficiency syndrome in 363 cases of IgA nephropathy. Zhongguo Zhong Xi Yi Jie He Shen Bing Za Zhi 2008; 05: 426-9.

15. Nie LF, Xu JL, Yu RH,Yu DJ, Sun HY, Han DY, et al. Overview of clinical practice guidelines for IgA nephropathy. Zhongguo Zhong Xi Yi Jie He
Shen Bing Za Zhi 2013; 14: 565-7.

16. Nie LF, Han DY, Yu DJ, Wang HX, Yu RH, Sun HY. Study on TCM syndrome distribution of 467 cases of chronic persistent IgA nephropathy. Zhongguo Zhong Xi Yi Jie He Shen Bing Za Zhi 2007; 8: 404-5.

17. Chen XM, Chen YP, Li P, Chen YP, Zhou ZL, Chen W, et al. A MultIcenteric Epidemiological Survey on TCM Syndrome in 1,016 Patients with IgA Nephropathy and Analysis of Its Relevant Factors. Zhongguo Zhong Xi Yi Jie He Za Zhi 2006; 26: 197-201.

18. Wang YJ, Chen HY, Zhu CF, Cheng XX, Zhang MO, Fang YQ, et al. Study on Syndrome-Manifestation of Traditional Chinese Medicine in 1148 Patients with IgA Nephropathy. Zhongguo Zhong Xi Yi Jie He Shen Bing Za Zhi 2009; 10: 1054-8.

19. Gu YH, Wang Y, Ji CL, Fan P, He ZR, Wang T, et al. Syndrome Differentiation of IgA Nephropathy Based on Clinicopathological Parameters: A Decision Tree Model. Evid Based Complement Alternat Med 2017; 2017: 2697560.

20. Liu LC, Yin ZW, Ma JW, Duan SW, Chen XM. Potential Association of Body Constitution with the Prognosis of IgA Nephropathy: A Long-Time Follow-Up of 203 Cases in China. Evid Based Complement Alternat Med 2019; 2019: 6289478.

21. Li JJ, Chen XM, Wei RB. Potential of Renal Pathology on Refining Syndrome Typing of Chinese Medicine in IgA Nephropath. Chin J Integr Med 2013; 19: 92-7.

22. Wang JZ, Liu P, He CY, Liu NA, Zhang PQ. Analysis on TCM syndromes and Patholgical Grading of 488 Patients with lga Nephrolmthy. Shi Yong Zhong Xi Yi Jie He Lin Chuang 2015; 15: 1-7.

23. Suzuki H, Kiryluk K, Novak J, Moldoveanu Z, Herr AB, Renfrow MB, et al. The pathophysiology of IgA nephropathy. J Am Soc Nephrol 2011: 22: 1795-803.

24. Li H, Yang XX, Yao GD, Zhang YX, Xu YY, Cao Y, et al. Triptolide inhibits tonsillar IgA production by upregulating FDC-SP in IgA nephropathy. Histol Histopathol 2019; 35: 599-608.

25. Nagayama Y, Nishiwaki H, Hasegawa T, Komukai D, Kawashima E, Takayasu M, et al. Impact of the new risk stratification in the 2011 Japanese society of nephrology clinical guidelines for IgA nephropathy on incidence of early clinical remission with tonsillectomy plus steroid pulse therapy. Clin Exp Nephrol 2015; 19: 646-52.

26. Ohya M, Otani H, Minami Y, Yamanaka S, Mima T, Negi S, et al. Tonsillectomy with steroid pulse therapy has more effect on the relapse rate than steroid pulse monotherapy in IgA nephropathy patients. Clin Nephrol 2013; 80: 47-52.

27. Ochi A, Moriyama T, Takei T, Uchida K, Nitta K. Comparison between steroid pulse therapy alone and in combination with tonsillectomy for IgA nephropathy. Inter Urol Nephrol: 2013; 45: 469-476.

28. Maeda I, Hayashi T, Sato KK, Shibata MO, Hamada M, Kishida M, et al. Tonsillectomy has beneficial effects on remission and progression of IgA nephropathy independent of steroid therapy. Nephrol Dial Transplant 2012; 27: 2806-13.

29. He Y, Yu RH, Wang T, Chen XJ. Investigation of mucosal involvement in 108 patients with IgA nephropathy. Zhongguo Zhong Xi Yi Jie He Shen Bing Za Zhi 2013; 14: 1095-6.

30. Jiang J, Wang XX, Shen PC, Sun C, He LQ. Clinical investigation of mucosal immune system in IgA nephropathy patients. DaLian Yi Ke Da Xue Xue Bao 2016; 38: 558-61.

31. Nie LF, Yu RH, Yu DJ, Fang JA, Zhang SR, He LQ, et al. The Efficacy of Yiqizishen Capsule in the Treatment of Hematuria in IgA Nephropathy. Zhongguo Zhong Xi Yi Jie He Shen Bing Za Zhi 2006; 4: 215-8.

32. Yu RH, Nie LF, Xu JL, Yu DJ, Han DY, Sun HY, et al. Clinical effect of Yiqi Zishen Granule on proteinuria in IgA nephropathy. Zhongguo Zhong Xi Yi Jie He Shen Bing Za Zhi 2010; 11: 721-2.

33. Chen XM, Chen J, Chen YP, Zhou ZL, He YN, Li P, et al. Multi center randomized controlled clinical observation of Shenhua Tablet in the treatment of IgA nephropathy (Qi and yin deficiency syndrome). Zhongguo Zhong Xi Yi Jie He Za Zhi 2007; 02: 101-5.

34. Li WW, Huang D, Shen PC, He LQ. Clinical Observation of Yiqi Guben Tiaomian Decoction in Throat-oriented Treatment of IgA Nephropathy. Zhongguo Shi Yan Fang Ji Xue Za Zhi 2016; 22: 166-70.

35. Wang XH, Liang Y, Yu RH, Liu J, Zhang RR, Xu JL, et al. Clinical study of 
Yiqi Yangyin Liyan decoction combined with pharyngeal pecking therapy in the treatment of IgA nephropathy with pharyngeal inflammation. Zhongguo Zhong Xi Yi Jie He Za Zhi 2019; 39: 52-6.

36. Wang QQ, Lu Y, Tang Y, Deng YY, Gao JD, Chen Y, et al. Treatment Outcome of TCM and WM Therapy on IgA Nephropathy with Chaiqin Decoction. Zhongguo Zhong Xi Yi Jie He Shen Bing Za Zhi 2020; 21: 309-12.

37. Chen HY, Ma HZ, Fu XJ, Guo LZ, Shen H, Huang WX, et al. IgA nephropathy treated from wind-damp syndrome: A multicenter, randomized, double blind, controlled trial study. Zhonghua Zhong Yi Yao Za Zhi 2018; 33: 1184-8.

38. Li J, Yu DR, Chen HY, Zhu CF, Cheng XX, Wang YH, et al. Long-term effect of the treatment of IgA nephropathy by tonifying shen, activating blood stasis, dispelling wind-dampness combined with western medicine. Zhongguo Zhong Xi Yi Jie He Za Zhi 2017; 37: 28-33.

39. Li PKT, Ho KKL, Szeto1 CC, Yu LM, Lai FMM. Prognostic indicators of IgA nephropathy in the Chinese-clinical and pathological perspectives. Nephrol Dial Transplant 2002; 17: 64-9.

40. Wang J, Zhang XX, Chen XN, Lu JR, Wang CH, Hu J, et al. A prospective multicenter clinical study of TCM syndrome differentiation combined with losartan potassium in the treatment of severe IgA nephropathy of liver and kidney yin deficiency type. Zhong Yi Za Zhi 2019; 60: 1929-34.

41. Chen WJ, Deng YY, Ni ZH, Wang NS, Wang Y, Deng W. Treatment of Zibuganshen Granules Plus Prednisone on Patients with Severe IgA Nephropathy (Ying-deficiency of the Liver and Kidney pattern): a Randomized, Double-blind, Placebo-controlled Multicentre Clinical Trial. Zhongguo Zhong Xi Yi Jie He Shen Bing Za Zhi 2015; 16: 405-9.

42. Chen WJ, Deng YY, Ni ZH, Wang NS, Wang Y, Deng W. Treatment of patients with severe IgA nephropathy (Pishenyangxu pattern) with Jianpibushentongluogranules plus prednisone: a randomized, controlled, and double-blind multicentre trial. Zhonghua Shen Bing Yan Jiu Dian Zi Za Zhi 2013; 2: 254-9.

43. Zhang W, Cheng C, Han Q, Chen Y, Guo J, Wu QN, et al. Flos Abelmoschus manihot extract attenuates DSS-induced colitis by regulating gut microbiota and Th17/Treg balance. Biomed Pharmacother 2019; 117: 109162.

44. Zhang L, Li P, Xing CY, Zhao JY, He YN, Wang JQ, et al. Efficacy and safety of Abelmoschus manihot for primary glomerular disease: a prospective, multicenter randomized controlled clinical trial. Am J Kidney Dis 2014; 64: 57-65.

45. Li P, Lin HL, Ni ZH, Zhan YL, He YN, Yang HT, et al. Efficacy and safety of Abelmoschus manihot for IgA nephropathy: A multicenter randomized clinical trial. Phytomedicine 2020; 76: 153231.

46. Wang Q, Xiao B, Cui SQ, Song HL, Qian YJ, Dong L, et al. Triptolide treatment reduces Alzheimer's disease (AD)-like pathology through inhibition of BACE1 in a transgenic mouse model of AD. Dis Model Mech 2014; 7: 1385-95.

47. Wang Z, Yu C, Zhou LN, Chen X. Effects of Tripterygium wilfordii Induction Therapy to IgA Nephropathy Patients with Heavy Proteinuria. Biol Pharm Bull 2017; 40: 1833-8.

48. Wyatt RJ, Julian BA. IgA nephropathy. N Engl J Med 2013; 368: 2402-14.

49. Meng HX, Ohtake H, Ishida A, Ohta N, Kakehata S, Yamakawa M. IgA production and tonsillar focal infection in IgA nephropathy. J Clin Exp Hematop 2012; 52: 161-70区

50. Barratt J, Eitner F, Feehally J, Floege J. Immune complex formation in IgA nephropathy: a case of the 'right' antibodies in the 'wrong' place at the 'wrong' time? Nephrol Dial Transplant 2009; 24: 3620-3.

51. Batra A, Smith AC, Feehally J, Barratt J. T-cell homing receptor expression in IgA nephropathy. Nephrol Dial Transplant 2007; 22: 2540-8.

52. Sun ZG, Hu YZ, Wang YG, Feng J, Dou YQ. BBupi Hewei decoction ameliorates 5-fluorouracil-induced intestinal dysbiosis in rats through Thelper 17/T regulatory cell signaling pathway. J Tradit Chin Med 2020; 40: 38-48.

53. Zhang YD, Biao YN, Chu XQ, Hao L, Shi C, et al. Protective effect of Chushizi (Fructus Broussonetiae) on acetaminophen-induced rat hepatitis by inhibiting the Toll-like receptor $3 / \mathrm{c}$-Jun $\mathrm{N}$-terminal kinase/cjun/c-fos/janus protein tyrosine kinase/activators of transcription 3 pathway. J Tradit Chin Med 2020; 40: 965-73.
54. Lv QW, Zhang W, Shi Q, Zheng WJ, Li X, Chen H, et al. Comparison of Tripterygium wilfordii HOOK F with methotrexate in the treatment of active rheumatoid arthritis (TRIFRA): a randomised, controlled clinical trial. Ann Rheum Dis 2015; 74: 1078-86.

55. Ren RY, Han Xue, Song CD. Effect of Tripterygium wilfordii multiglucoside on intestinal flora and immune function in IgA nephropathy rats based on C1GALT1/Cosmc pathway. Zhongguo Bing Li Sheng Li Za Zhi 2020; 36: 2050-5.

56. Chen MZ, Li Y, He W. Effect of Hesperidin on STAT3 and p-JAK2 Protein Expressions Related to JAK/STAT Signal Pathway in Renal Tissues of IgA Nephropathy Rats. Jie Fang Jun Yi Yao Za Zhi 2017; 29: 10-4.

57. Chen XW, Peng SN, Zeng HH, Fu AX, Zhu QX. Toll-like receptor 4 is involved in a protective effect of rhein on immunoglobulin A nephropathy. Indian J Physiol Pharmacol 2015; 47: 27-33.

58. Liang Y, Wang XH, Liu J, Xu JL, Zhang RR, Li L. Effect of Yiqi Yangyin Liyan decoction combined with pharyngeal pecking therapy on lymphocyte subsets of 43 cases of IgA nephropathy with pharyngeal inflammation. Beijing Zhong Yi Yao 2019; 38: 809-12.

59. Zou D, Zhang SL, Wang YP, Wang HA. Influences of Gouqi Fushen pills for Th1/Th2 of IgA nephropathy model rats. Shizhen Guo Yi Guo Yao 2020; 31: 257-9.

60. Zhang RC, Zhong LB, Zhou JF, Peng YM. Complement-C1q TNF-related protein 3 all eviates mesangial cell activation and Inflammatory response stimulated by secretory IgA. Am J Nephrol 2016; 43: 460-8.

61. Li HL, Lu RR, Pang Y, Li JC, Cao YW, Fu HX, et al. Zhen-Wu-Tang Protects IgA Nephropathy in Rats by Regulating Exosomes to Inhibit NF-kB/NLRP3 Pathway. Front Pharmacol 2020; 16: 1080.

62. Liu BH, Lu RR, Li HG, Zhou Y, Zhang PC, Bai LX, et al. Zhen-wu-tang ameliorates membranous nephropathy rats through inhibiting NF-kB pathway and NLRP3 inflammasome. Phytomedicine 2019; 59: 152913.

63. Yang L, Wang Y, Nuerbiye A, Cheng P, Wang JH, Kasimu R, et al. Effects of Periostracum Cicadae on Cytokines and Apoptosis Regulatory Proteins in an IgA Nephropathy Rat Model. Int J Mol Sci 2018; 19: 19061599.

64. Deng F, Zhang JW, Li Y, Wang W, Hong DQ, Li GS. Hirudin ameliorates immunoglobulin A nephropathy by inhibition of fibrosis and inflammatory response. Renal Failure 2019; 41: 1583113.

65. Qin JH, Lin JR, Ding WF, Wu WH. Schisandrin B Improves the Renal Function of IgA Nephropathy Rats Through Inhibition of the NF-kB Signalling Pathway. Inflammation 2019; 42: 884-94.

66. Wen Q, Yan L, Zhou JB, Zhao CH, Zhang J, Shan K, et al. TSP-1 promotes glomerular mesangial cell proliferation and extracellular matrix secretion in Thy-1 nephritis rats. J Biomed Res 2011; 25: 402-10.

67. Zu N, Li P, Li N, Choy P, Gong Y. Mechanism of saikosaponin-d in the regulation of rat mesangial cell proliferation and synthesis of extracellular matrix proteins. Biochem Cell Biol 2007; 85: 169-74.

68. Zeng JL, Jiang X, Yu J, Tang XL, Lin X, Ge SS. Renal protection effect of kunxian capsule on rat with IgA nephropathy and its influence on mesangial cell proliferation. Zhongguo Xian Dai Yi Sheng 2019; 57: 38-43.

69. Chen HY, Du YY, Li YY, Zeng JL, Miao JX, Jiang X. Jixuecao (Herba Centellae Asiaticae) alleviates mesangial cell proliferation in $\operatorname{IgA}$ nephropathy by inducing mitofusin 2 expression. J Tradit Chin Med 2019; 39: 346-55.

70. Wan Y, Sun W, Zhang H, Yan Q, Chen P, Dou C, et al. Multi-glycoside of Tripterygium wilfordii Hook. F. ameliorates prolonged mesangial lesions in experimental progressive glomerulone phritis. Nephron Exp Nephrol 2010; 114: 7-14.

71. Zhang G, Chen JH, Liu Y, Yang R, Guo HQ, Sun ZY. Triptolideconditioned dendritic cells induce allospecific T-cell regulation and prolong renal graft survival. J Invest Surg 2013; 26: 191-9.

72. Chi X, Zhang H, Zhang S, Ma K. Chinese herbal medicine for gout: a review of the clinical evidence and pharmacological mechanisms. Chin Med 2020; 15: 17.

How to cite this article: Wang XH, Lang R, Liang Y, Zeng Q, Chen N, Yu $\mathrm{RH}$. Traditional chinese medicine in treating IgA nephropathy: from basic science to clinical research. J Transl Intern Med 2021; 9: 161-7. 